\title{
EGFR and c-Met Inhibitors are Effective in Reducing Tumorigenicity in Cancer
}

\author{
Amanda Stone $^{1 \#,}$ Kymberly Harrington ${ }^{1}$, Mark Frakes ${ }^{2}$, Kory Blank ${ }^{2}$, Supriya Rajanna ${ }^{1}$, Ichwaku Rastogi ${ }^{1}$ and Neelu Puri $^{{ }^{* \#}}$ \\ ${ }^{1}$ Department of Biomedical Sciences, University of Illinois at Rockford, Rockford, USA \\ ${ }^{2}$ University of Illinois at Rockford, College of Medicine at Rockford, Rockford, USA \\ \#Both authors have contributed equally to this work \\ *Corresponding author: Neelu Puri, Department of Biomedical Sciences, University of Illinois College of Medicine at Rockford, 1601 Parkview Avenue, Rockford, \\ Illinois 61107, USA,, Tel: 815-395-5678; Fax: 815-395-5666; E-mail: neelupur@uic.edu
}

Received date: Mar 31, 2014, Accepted date: Apr 28, 2014, Published date: May 5, 2014

Copyright: $\odot 2014$ Stone A, et al. This is an open-access article distributed under the terms of the Creative Commons Attribution License, which permits unrestricted use, distribution, and reproduction in any medium, provided the original author and source are credited.

\begin{abstract}
EGFR and c-Met are receptor tyrosine kinases that are implicated in tumor development and progression in several types of cancer. Both EGFR and c-Met, which are known to be overexpressed and mutated in cancer, share common signaling pathways, including the PI3K/ Akt and MAPK pathways. Small molecule tyrosine kinase inhibitors and monoclonal antibodies that work against EGFR and c-Met are at the forefront of cancer therapy, but their individual efficacies are limited due to the development of drug resistance. In recent pre-clinical studies, we observed that combination therapy using mTOR and Wnt inhibitors with EGFR or c-Met tyrosine kinase inhibitors resulted in overcoming drug resistance. Our studies also indicated that EGFR and c-Met tyrosine kinase inhibitor resistance may be due to activation of alternative signaling pathways. The development of additional combinatorial therapies is underway, and various combinations of inhibitors have shown promising results in clinical trials. Future studies in this direction could be the basis for development of new cancer therapeutics, utilizing EGFR and c-Met inhibitors, which could greatly improve patient prognosis.
\end{abstract}

Keywords: EGFR; c-Met; Tyrosine kinase inhibitor; Monoclonal antibody; Resistance

\section{Introduction}

In 2014, an estimated $1,665,540$ total cancer diagnoses and 585,720 cancer mortalities will occur in the United States. During recent years, cancer incidence rates have declined in men by $0.6 \%$ per year, while incidence rates have remained stable in the female population. Cancer mortality rates have decreased in males by $1.8 \%$ per year and $1.4 \%$ per year in females [1]. In less developed countries located in South America, Africa, and Asia, the incidence and mortality rates of most cancers are on the rise, most likely due to incorporation of unhealthy western lifestyles, such as smoking, high fat diet, and consumption of highly processed foods [2]. The most frequent cancer diagnosis in the United States, in males, is in the genitourinary system $(243,460)$, which is primarily due to the large number of prostate cancer diagnoses $(233,000)$. In the female population, breast malignancies are the most frequent cancer diagnoses $(232,670)$. However, lung and bronchus cancers are estimated to have the highest mortality of all malignancies, for both sexes, in $2014(163,660)$ [1].

Human epidermal growth factor receptor (EGFR) and c-Met are receptor tyrosine kinases (RTKs) that are encoded by genes located on the short arm of chromosome $7[3,4]$. Both RTKs have an extracellular ligand binding domain, a transmembrane portion, and a tyrosine kinase moiety, located intracellularly [4,5]. Under physiological conditions, EGFR is activated by numerous ligands, including epidermal growth factor (EGF). The activated receptor becomes bound to either an identical receptor (homodimerization) or another receptor in the HER/ErbB family (heterodimerization) [6]. In contrast, c-Met is activated by a single ligand, known as hepatocyte growth factor/scatter factor (HGF), which is secreted by fibroblasts, smooth muscle cells, and other mesenchymal cells [4]. Stimulation of EGFR and c-Met, by binding of their corresponding ligands, results in autophosphorylation of the tyrosine kinase domains, which causes downstream signaling and further activation of the mitogen-activated protein kinase (MAPK), RAS-RAF-MEK-ERK/MAPK, and phosphatidylinositol 3-kinase (PI3K)/Akt pathways [3,7] (Figure 1). Under physiological conditions, the PI3K/Akt and MAPK pathways are involved in diverse cellular processes, such as angiogenesis, metabolism, growth, proliferation, survival, protein synthesis, transcription, apoptosis [8], differentiation, organism development, and cell cycle regulation and progression $[9,10]$.

Cancer cells, namely squamous cell carcinomas of the head and neck, colorectal cancer (CRC), glioblastoma, and non-small cell lung cancer (NSCLC), express RTKs such as EGFR and c-Met [11,12]. However, RTKs on tumor cells have altered functions due to mutations or overexpression, which can result in increased activation and tumorigenesis. Inhibition of these altered tyrosine kinases has become a therapeutic strategy for patients with tumors that overexpress these receptors or contain mutated forms of these RTKs. Inhibition methods and therapeutic options include molecules that inhibit tyrosine kinase activity, and monoclonal antibodies (MAbs) that interfere with ligand binding to RTKs [11]. MAbs, such as cetuximab (EGFR inhibitor), and small molecule tyrosine kinase inhibitors (TKIs), such as gefitinib, erlotinib (EGFR inhibitors) and crizotinib (c-Met/ALK/ROS1 inhibitor), are currently used for NSCLC therapy and have shown great promise in improving prognosis of several other forms of cancer [12-14] (Figure 1). 


\section{EGFR: Structure, Targeted Pathways and Dysregulation in Cancer}

EGFR is a member of the HER/ErbB RTK family, which consists of four proteins, EGFR (ErbB1, Her1), HER2 (EGFR2, Her2/neu), HER3 (EGFR3, ErbB3), and HER4 (EGFR4 or ErbB4) [13,15]. The general structure of this family of receptors is made up of a glycosylated extracellular domain, a hydrophobic transmembrane portion, and an intracellular section with a juxtamembrane (JM) segment, a protein kinase domain, and a carboxyterminal tail $[5,16]$. The extracellular domain contains four parts, which include leucine-rich segments that are known to participate in ligand binding (domains I and III) and sectors with numerous cysteine residues that are involved in disulfide bond formation (domains II and IV) [5,17]. In addition, domain II participates in homodimer and heterodimer formation with HER/ ErbB family members. The ability of these proteins to function as homodimers and heterodimers, in various combinations, allows EGFR to initiate complex signaling cascades [16]. To add another layer of complexity, there are numerous known ligands (greater than 30) [18] for the HER/ErbB family, out of which there are seven ligands that bind to EGFR specifically, including EGF, transforming growth factoralpha, heparin-binding EGF-like growth factor, amphiregulin, betacellulin, epiregulin, and epigen [19]. It has also been found that the binding of particular ligands can cause induction of diverse dimerization reactions, such as binding of EGF, which induces heterodimerization of EGFR with HER2, HER3 or HER4 [20].

In regards to cancer therapeutics, EGFR and its family members have emerged as promising targets, due to their multiple and diverse roles in cancer progression, as well as their overexpression or mutation status in several types of cancers, including lung, breast, pancreatic, esophageal, CRC, and head and neck cancer [16,21-23]. Specifically, EGFR has been demonstrated to play an important role in the development and growth of tumor cells, in which it is involved in multiple cellular responses, such as apoptosis and proliferation [24,25]. Overexpression of EGFR has been implicated in greater than $60 \%$ of NSCLC patients. NSCLC cell lines also produce EGFR ligands, EGF and transforming growth factor-alpha, which bind to the EGFR receptors in an autocrine fashion, resulting in cell proliferation and gene transcription [3]. EGFR mediates these cellular responses via several downstream signaling pathways, including the PI3K/Akt and the MAPK pathways [26]. The PI3K/Akt pathway is known to have an important role in mediating cell survival, while the MAPK pathway is associated with cell proliferation [8-10]. These pathways and other HER/ErbB family signaling components further regulate angiogenesis, adhesion, cell motility and migration, development, differentiation, organogenesis, and survival of cancer cells $[5,16,18]$.

\section{c-Met: Structure, Targeted Pathways and Dysregulation in Cancer}

c-Met is a disulfide-linked heterodimer that is comprised of a 50 $\mathrm{kDa} \alpha$-chain and a $140 \mathrm{kDa} \beta$-chain $[7,14,27]$. The extracellular region of c-Met is composed of a seven-bladed $\beta$-propeller semaphorin (sema) domain, which is attached to a PSI domain. The PSI domain is subsequently attached to four immunoglobulin-like repeat domains. In addition, the sema domain contains the binding site for HGF, the only known naturally occurring stimulating factor of c-Met [14,27]. HGF is a six domain signaling peptide originally identified as a growth factor for hepatocytes and as a motogenic factor for fibroblasts [14]. The six domains of HGF are comprised of one N-terminal domain, four kringle domains, and a C -terminal domain [14]. HGF binds to the sema domain, which leads to c-Met dimerization and intrinsic tyrosine kinase activation due to the phosphorylation of tyrosine residues, Y1230, Y1234, and Y1235, within the intracellular tyrosine kinase domain [4]. In addition, the intracellular region of c- Met is composed of a JM domain and a carboxyterminal tail, which is believed to be involved in downstream signaling [27]. The tyrosine kinase region is also responsible for the initiation of downstream signaling, primarily through the PI3K/Akt, MAPK, and signal transducers and activators of transcription (STAT) pathways [7]. Activation of these pathways is necessary for cell scattering, a crucial step in wound healing and tissue repair and reconstruction [7]. PI3K is also required for c-Met and HGF mediated mitogenesis and motogenesis [14]

Concerning dysregulation in cancer, c-Met is frequently associated with tumor growth, invasion and metastasis [7,27,28]. Cancer cells are able to gain malignancy through overexpression of the c-Met receptor and HGF, autocrine signaling, mutations leading to increased receptor activation, and gene amplification [27]. Furthermore, studies have shown correlations between tumor type and specific dysregulation. Autocrine stimulation of c-Met is most commonly seen in gliomas, osteosarcoma, pancreatic cancers, and gastric cancers [28]. Signaling associated with c-Met mutations leads to increased angiogenesis, tumor invasion, metastasis, and decreased overall prognosis of cancers [28]. c-Met is also frequently co-expressed with PAX5, a nuclear transcription factor in small cell lung cancer (SCLC) and neuroendocrine tumors $[14,29]$. Moreover, one study proposed c-Met as a target of the Wnt signaling cascade, which initiates CRC tumorigenesis [30].

\section{Mutations in EGFR and MET}

Activating mutations in EGFR and c-Met RTKs are linked to the formation of tumors in multiple types of cancers. Mutations that involve the kinase domain of EGFR are labelled as activating mutations, due to activation of tyrosine kinase activity that is independent of ligand binding [31]. This region also serves as the binding site for various small molecule inhibitors, such as erlotinib and gefitinib [31]. Mutations of the EGFR gene are located in the first four exons of the tyrosine kinase domain (18 through 21). Activating somatic mutations of EGFR fall within three classes. Class I mutations are in-frame deletions in exon 19, which result in the loss of four to six amino acids (E746 to S752). Class II mutations consist of single nucleotide substitutions, occurring in exons 18 through 21, which alter the amino acid sequence. Lastly, class III mutations are in-frame duplications or insertions of exon 20 [32]. The majority of tyrosine kinase domain mutations occur in either exon 19, including class I deletions, or exon 21, such as missense mutations at 858 (L858R) in which arginine is substituted for leucine [32]. A recent discovery has also revealed a rare exon 22 mutation (E884K) of EGFR that works in concert with L858R to alter sensitivity to EGFR kinase inhibitors. The presence of both E884K and L858R has been shown to decrease erlotinib inhibition, however, these mutations together increase inhibition by gefitinib [33]. Overall, EGFR mutations within the tyrosine kinase domain destabilize the conformation of the domain and lead to prolonged activation of kinase function, as well as downstream signaling pathways [31].

The threonine-790 to methionine (T790M) germline mutation is a specific alteration of the EGFR exon 20 segment. T790M is characterized as a secondary point mutation due to its presence in 
Citation: Stone A, Harrington K, Frakes M, Blank K, Rajanna S (2014) EGFR and c-Met Inhibitors are Effective in Reducing Tumorigenicity in

Page 3 of 9

patients who were initially responsive to EGFR TKIs, but gradually developed resistance to this treatment [31]. The T790M point mutation can be found near the kinase active site and is associated with resistance to gefitinib-induced suppression of EGFR activation through increased ATP affinity of the receptor, which reduces TKI efficacy [31]. Specifically in NSCLC, acquisition of T790M accounts for more than $50 \%$ of all acquired resistance against EGFR TKIs in patients [31,34], and the presence of this mutation prior to first-line treatment with TKIs has been shown to result in the development of primary resistance $[35,36]$. Additional secondary point mutations include T854A in exon 21, and the L747S and D761Y mutations of exon 19 [32].

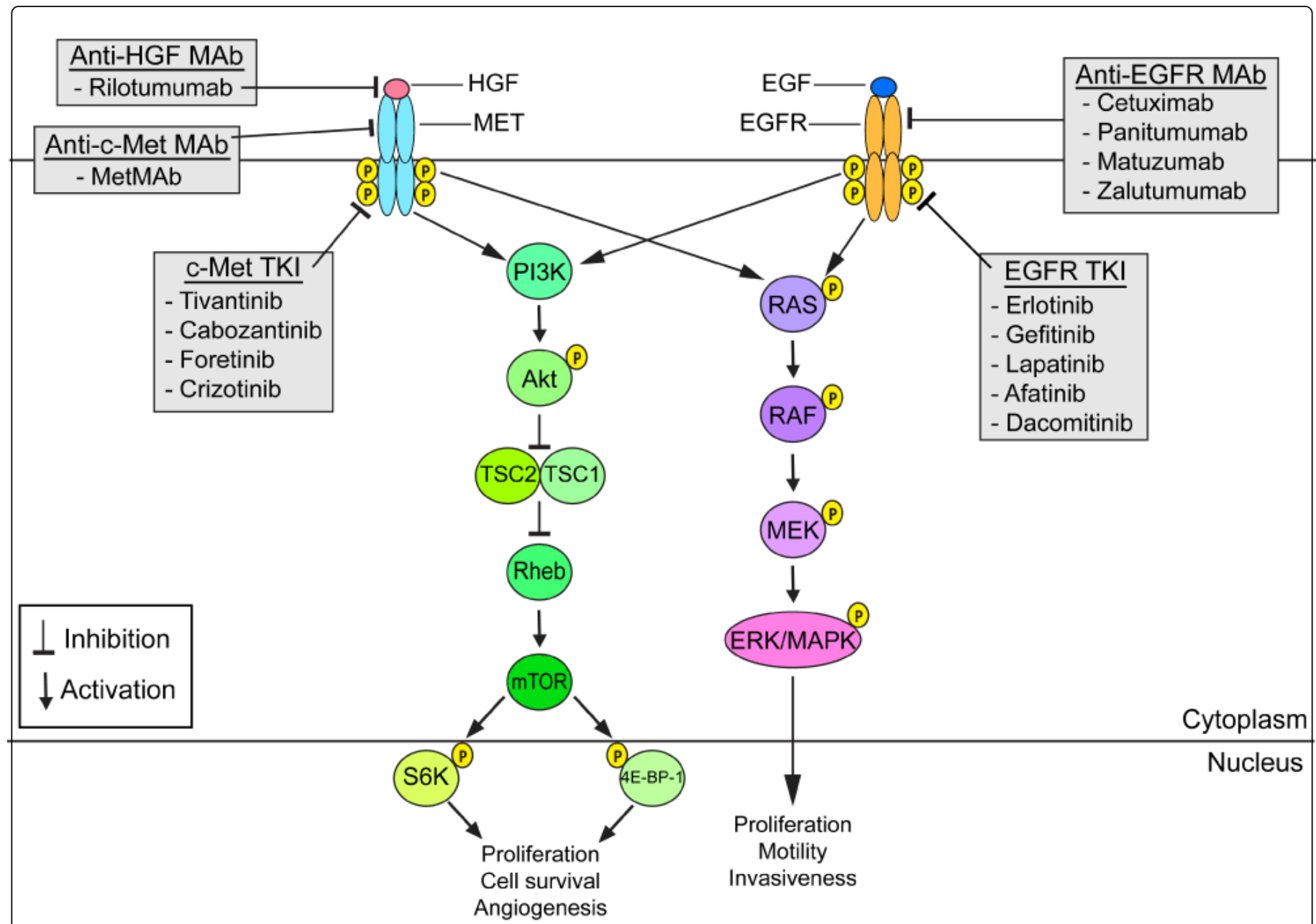

Figure 1: Effect of inhibitors on EGFR and c-Met signaling pathways in cancer: The binding of ligands EGF/HGF to their corresponding receptors, EGFR/c-Met, leads to phosphorylation of specific tyrosine residues and activation of these receptors. Overexpression of EGFR and c-Met RTKs results in activation of downstream signaling pathways, PI3K/Akt and MAPK (RAS-RAF-MEK-ERK/MAPK), in several cancers. The induction of these signaling cascades results in the stimulation of cancer cell survival through dysregulation of cell death pathways. Several inhibitors are in clinical trials that inhibit these pathways, by binding to tyrosine kinase domains or to ligands, resulting in receptor inactivation. TKIs and MAbs of the EGFR/c-Met signaling pathways are shown in gray boxes, with their targets marked by inhibitory arrows.

Overexpression of MET can be due to transcriptional activation, hypoxia or amplification, which has been associated with metastasis of primary tumors and a poor prognosis in patients with breast, endometrial, hepatocellular, and renal carcinomas, as well as gastric, CRC and bladder cancers [37]. MET mutations may activate the receptor through high-affinity binding of the HGF ligand or through autophosphorylation. Activation of the receptor induces a signal cascade, which results in subsequent activation of various signal transducers such as GRB2, GAB1, PI3K, STAT, ERK, and FAK. The MET gene also has activating mutations, particularly in the sema and JM domains. Mutations in the MET gene, T1010I and R988C, increase the rate of phosphorylation of the signal transducer and adaptor protein, paxillin, which is also overexpressed in NSCLC [14]. Under normal conditions, c -Met signaling and activation by HGF can be terminated by c-CBL, an E3 ubiquitin ligase, which binds to Y1003 on the JM domain. This c-CBL binding leads to the internalization of the c-Met receptor via clathrin-coated vesicles [14]. However, when the Y1003 position is mutated, the HGF receptor is not ubiquitinated, resulting in decreased lysosomal degradation, increased stability and additional oncogenic activation [38]. Mutations of the JM domain are associated with SCLC (S1040P, T992I, and R970C) as well as other cancers including melanoma (N930S) and gastric carcinoma (P991S) [14]. 
Missense mutations that occur in MET can be found within the tyrosine kinase domain. The formation of solid tumors due to these missense mutations occurs in hereditary papillary renal carcinomas (HPRC), childhood hepatocellular carcinoma, and a small percentage of squamous cell carcinomas of the head and neck, leading to the invasion and metastasis of tumor cells [39]. MET missense mutations, R970C and T992I, are also found in nearly 3\% of CRC [40]. These mutations disrupt phosphorylation of the serine residue, S985, which normally negatively regulates c-Met kinase activity. Missense mutations of MET in HPRC can positively regulate c-Met kinase activity when the mutation occurs on either side of two tyrosine residues at the catalytic sites, pY1234 and pY1235. The variation between mutational activities in CRC versus HPRC patients is thought to be a result of negative or positive activation of the c-Met kinase [40]. The sema domains are necessary for the dimerization, activation, and regulation of c-Met, and may be targeted for therapeutic treatment in c-Met dependent cancers that are regulated by activating mutations of MET. Mutations in the sema domain are often seen in lung cancers and nearly $7 \%$ of mesothelioma patients (N375S, M431V, and N454I) [38]. However, additional research is needed to unveil the exact functional role of the various MET mutations mentioned above.

\section{EGFR and c-Met as Therapeutic Targets: Agents targeting EGFR and c-Met}

EGFR and c-Met are considered as important molecular targets in current cancer therapeutics. The most common therapies targeting EGFR and c- Met are kinase inhibitors and MAbs [3,41]. TKIs are small-molecule kinase inhibitors that bind to the intracellular domain of RTKs and inhibit their function. They can be classified by their mechanism of action, in which they compete with adenosine triphosphate (ATP) or act as allosteric molecules. TKIs can be further classified by their binding capabilities as reversible or irreversible inhibitors [42]. Most MAbs work by binding to the extracellular domain of tyrosine kinases and inhibit the binding of receptor ligands, hence inhibiting receptor activation [43]. Cancer cells often amplify and overexpress growth factor receptors, EGFR and c-Met, in order to proliferate [44]. Therefore, MAbs against EGFR and c-Met receptors are used to specifically target cancer cells and to inhibit proliferation by preventing binding of their ligands, EGF and HGF, respectively. Several TKIs and MAbs have been designed against EGFR and c-Met receptors, some of which are currently being used as first-line cancer therapies and others that have advanced into clinical trials [37] (Table $1)$.

\section{EGFR TKIs and MAbs}

EGFR TKIs act by binding to the ATP binding site of EGFR, which inhibits receptor phosphorylation and hence downstream signaling. Erlotinib and gefitinib are reversible TKIs, which have been approved for the treatment of NSCLC patients [3]. Lapatinib is a dual kinase inhibitor that targets EGFR, as well as the HER2 receptor [45]. Although lapatinib is under late phases of clinical trials for NSCLC (NCT01306045, NCT00313599), it is currently used for treatment of patients with HER2 receptor positive breast cancer in combination with capecitabine [5]. Since reversible TKIs acquire resistance over time, irreversible TKIs that bind covalently to the kinase domain of EGFR and inhibit phosphorylation of EGFR irreversibly are being developed. Afatinib and dacomitinib are examples of such second generation irreversible TKIs. Afatinib has been approved for clinical use in adult patients with advanced or metastatic NSCLC with L858R mutations [46] and dacomitinib is in late stages of clinical trials [47]. In regards to MAbs, anti-EGFR MAbs function as competitive antagonists by blocking EGF binding, hence inhibiting the function of EGFR [48]. Cetuximab, panitumumab, matuzumab, and zalutumumab are a few examples of MAbs that work against EGFR [48-50]. Cetuximab, a chimeric human/murine IgG1 MAb, and panitumumab, a fully humanized IgG2 MAb, are the only monoclonal antibodies that are approved for the treatment of metastatic CRC, and the remaining MAbs mentioned above are currently in clinical trials $[45,48]$ (Table 1) (Figure 1).

\section{c-Met TKIs and MAbs}

Tivantinib, cabozantinib, foretinib and crizotinib are c-Met TKIs that have shown in clinical trials [37,51-54]. Tivantinib (ARQ197) is a non-ATP competitive c-Met TKI that disrupts phosphorylation of , resulting in inhibition of c-Met downstream signaling. However, recent studies indicate that it may also disrupt microtubule formation $[55,56]$. Cabozantinib and foretinib are both multikinase inhibitors, while crizotinib inhibits c-Met as well as ALK and ROS1. Furthermore, cabozantinib is the only c- Met TKI that has been approved by the FDA for the treatment of medullary thyroid cancer [57]. In addition to the above TKIs, anti-c-Met and HGF MAbs, such as MetMAb (OA-5D5) and rilotumumab, respectively, have shown some promising results in recent clinical trials. MetMAb, a monovalent antagonistic MAb, has shown increased overallsurvival in patients with high c-Met expression [37,58], and rilotumumab has been shown to inhibit further progression of various solid tumors and to decrease c-Met phosphorylation [59-61] (Table 1) (Figure 1).

\section{EGFR/c-Met Inhibitor Pre-Clinical Studies}

Various pre-clinical studies have been conducted, by our laboratory and others, to further investigate the efficacy of combination therapies with EGFR and c-Met inhibitors, as well as to evaluate mechanisms of resistance to EGFR/c-Met inhibition. Xu et al. tested the combination of crizotinib (PF2341066) and gefitinib in head and neck squamous cell carcinoma (HNSCC) cell lines to study the combined ability of inhibiting cell proliferation, invasion and wound healing. Various concentrations of the inhibitors were used, and overall, the combination treatment was observed to inhibit cell growth by $79.7 \%$ to 95.1\% in these cell lines. Moreover, the results demonstrated that the combination of these EGFR and c-Met inhibitors was more effective when compared to either inhibitor used as a single therapy alone [62]. Castoldi et al. developed a bispecific antibody, MetHer1, which was able to successfully target both EGFR and c-Met. MetHer1 was found to reduce proliferation in epidermoid carcinoma and NSCLC cancer cell lines, in an additive manner, compared to single and double drug treatments with cetuximab and 5D5.v2. This bispecific antibody was also shown to be 7 -fold more potent in inhibiting migration than a combination of cetuximab and 5D5.v2 at low concentrations. Furthermore, MetHer1 down regulated EGFR and c-Met downstream signaling proteins, such as p-ERK and p-Akt, in five cancer cell lines in vitro, and inhibited tumor growth in NSCLC xenografts in vivo [63].

Previous studies in our laboratory have demonstrated that combining ligands of EGFR and c-Met, EGF and HGF, resulted in synergistic activation and significant stimulatory effects on NSCLC cell proliferation, motility and downstream signaling [44]. Cross-talk between EGFR and c-Met has been further demonstrated by the fact that HGF can transactivate EGFR, which can phosphorylate and activate c-Met, resulting in synergistic effects on tumor development 
Page 5 of 9

and progression [64-66]. c-Met has also been identified as a key player in the development of resistance against EGFR inhibitors. Specifically, in NSCLC, $20 \%$ of acquired resistance to EGFR TKIs can be attributed to the amplification of the MET gene [67-69], most likely due to this signaling cross-talk between EGFR and c-Met RTKs [44]. In our more recent study, a resistant NSCLC cell line (H358) was treated with single, double, and triple combinations of EGFR (erlotinib), c-Met (SU11274), and mTOR (everolimus) inhibitors. The triple combination was found to inhibit H358 resistant cells by $95 \%$, in comparison to $34 \%$ inhibition after double combination therapy. In addition, treatment of XAV939, a Wnt inhibitor, combined with SU11274 and erlotinib, resulted in an 85\% decrease in cell viability in resistant H2170 NSCLC cells. Upregulation of downstream signaling proteins, including p-mTOR, p-p70S6K, p-4E-BP1, p-ERK, active $\beta$ catenin, p-LRP6 and Axin1, was also observed in H358 and H2170 NSCLC cell lines resistant to erlotinib and SU11274 [70].

Other studies have focused on microRNA (miRNA), small inhibitory non-coding RNA, dysregulation in the investigation of cancer development and resistance. A recent study found c-Met to regulate a miRNA cluster, miR-23a 27a 24-2, through ELK1, a transcription factor downstream of the MAPK pathway, which is activated by phosphorylated ERK [71,72]. miR-27a, a mature miRNA member of the miR-23a 27a 24-2 cluster, was also observed to subsequently downregulate endogenous proteins levels of EGFR and c-Met in NSCLC cells [72]. The study further proposed two mechanisms of miR-27a regulation of these RTKs, which included the direct targeting of c-Met and EGFR mRNA 3' UTRs and the indirect downregulation of Sprouty2, a MAPK pathway and signaling antagonist $[72,73]$.

\section{Clinical Trials}

\section{EGFR inhibitor clinical trials}

Current clinical trials are ongoing in order to overcome resistance due to secondary EGFR mutations, which have been observed in several types of cancer [74]. Therefore, targeting various downstream and parallel pathways to inhibit the activation of EGFR in cancer cells is thought to be more efficacious. Present clinical trials are also focusing on combinatorial therapies to improve the efficacy of antiEGFR treatments [74]. Clinical trials involving a combination of oral gemcitabine and erlotinib have been conducted to target EGFR. The phase III trial of this study resulted in significantly improved overall survival (OS) in patients with advanced pancreatic cancer following erlotinib and gemcitabine treatment, compared to treatment with gemcitabine alone [75]. The gemcitabine and erlotinib treatment group resulted in a $23 \%$ improvement in 1-year survival, and a similar result was found for OS. Furthermore, the observed improvement in progression-free survival (PFS) of this combination treatment supports the beneficial effects of erlotinib treatment in pancreatic cancer patients [75].

Another phase III clinical trial aimed at characterizing responses to gefitinib was carried out in 172 chemotherapy-naive NSCLC patients, age 75 or younger, positive for EGFR exon 19 deletions or L858R point mutations. In this study, gefitinib was administered orally every 21 days for three to six cycles. Gefitinib treatment showed a significantly longer PFS of 9.2 months, as compared to the cisplatin and docetaxel treatment group, which showed a PFS of 6.3 months [76]. In a recent update to this study OS was reported, which demonstrated a survival increase of 3 years for gefitinib treated NSCLC patients with EGFR mutations. As a second-line therapy, $61 \%$ of patients treated with gefitinib also received platinum doublet, however, the remaining $40 \%$ of patients, treated only with gefitinib, had similar outcomes as compared to patients treated using both methods [77] (Table 1).

\begin{tabular}{|c|c|c|c|c|}
\hline Compound & Targets & Study Phase & Monotherapy or Combination (C) & Tumor Type \\
\hline \multicolumn{5}{|c|}{ EGFR Inhibitors } \\
\hline \multirow[t]{3}{*}{ Erlotinib } & \multirow[t]{3}{*}{ EGFR TKI } & a) FDA & a) Monotherapy & a) NSCLC [5] \\
\hline & & b) FDA & b)C: Gemcitabine & b) Pancreatic cancer [5] \\
\hline & & c) III & c) C: Gemcitabine & c) Pancreatic cancer [75] \\
\hline \multirow[t]{2}{*}{ Gefitinib } & \multirow[t]{2}{*}{ EGFR TKI } & a) FDA* & a) Monotherapy & a) NSCLC [5] \\
\hline & & b) III & b) C: Cisplatin and Docetaxel & b) NSCLC $[76,77]$ \\
\hline \multirow[t]{2}{*}{ Lapatinib } & \multirow[t]{2}{*}{ EGFR, HER2 TKI } & a) FDA & a) C: apecitabine & a) HER2+ breast cancer [5] \\
\hline & & b) III & b) C: Paclitaxel & b) HER2+ metastatic breast cancer [50] \\
\hline \multirow[t]{2}{*}{ Afatinib } & \multirow[t]{2}{*}{ EGFR, HER2 TKI } & a) FDA & a) Monotherapy & a) NSCLC [5] \\
\hline & & b) III & b) Monotherapy & b) HNSCC (NCT01427478) \\
\hline \multirow[t]{2}{*}{ Dacomitinib } & \multirow[t]{2}{*}{ EGFR TKI (pan- HER inhibitor) } & a) II & a) C: Cetuximab & a) HNSCC [49] \\
\hline & & b) III & b) C: Gefitinib & b) NSCLC (NCT01774721) \\
\hline Cetuximab & EGFR MAb & a) FDA & a) C: Cytotoxic or radiation therapies & a) CRC, head and neck cancer [5] \\
\hline
\end{tabular}


Citation: Stone A, Harrington K, Frakes M, Blank K, Rajanna S (2014) EGFR and c-Met Inhibitors are Effective in Reducing Tumorigenicity in Cancer. J Carcinog Mutagen 5: 173. doi:10.4172/2157-2518.1000173

Page 6 of 9

\begin{tabular}{|c|c|c|c|c|}
\hline & & b) $I / / I I I$ & b) C: Platinum -based chemotherapy & b) NSCLC [86] \\
\hline \multirow[t]{2}{*}{ Panitumumab } & \multirow[t]{2}{*}{ EGFR MAb } & a) FDA & a) Monotherapy & a) CRC [5] \\
\hline & & b) III & b) C: FOLFOX regimen & b) Metastatic CRC (NCT00364013) \\
\hline Matuzumab & EGFR MAb & II & C: Pemetrexed & NSCLC (NCT00111839) \\
\hline Zalutumumab & EGFR MAb & III & C: Radiotherapy & HNSCC (NCT00496652) \\
\hline \multicolumn{5}{|c|}{ c-Met Inhibitors } \\
\hline \multirow[t]{3}{*}{ Tivantinib } & \multirow[t]{3}{*}{ c-Met TKI } & a.) I & a) C: Sorafenib & a) $\mathrm{HCC}$ [83] \\
\hline & & b.) II & b) C: Erlotinib & b) NSCLC [84] \\
\hline & & c.) III & c) Monotherapy & c) NSCLC, HCC (NCT01755767) \\
\hline \multirow[t]{3}{*}{ Cabozantinib } & \multirow[t]{3}{*}{ c-Met/multi-kinase TKI } & a) FDA & a) Monotherapy & a) Medullary thyroid cancer [28] \\
\hline & & b) II & b) Monotherapy & b) CRPC [80] \\
\hline & & c) III & c) C: Prednisone & c) CRPC (NCT01605227) \\
\hline Foretinib & c-Met/multi-kinase TKI & II & Monotherapy & Renal papillary cell carcinoma [87] \\
\hline \multirow[t]{2}{*}{ Crizotinib } & \multirow[t]{2}{*}{ c-Met, ALK, ROS1 TKI } & a) FDA & a) Monotherapy & a) ALK+ NSCLC [28] \\
\hline & & b) II & b.) Monotherapy & $\begin{array}{l}\text { b) Hematologic and metastatic cancers, } \\
\text { and solid tumors (NCT02034981) }\end{array}$ \\
\hline \multirow[t]{2}{*}{ MetMAb } & \multirow[t]{2}{*}{ c-Met MAb } & a) II & a) C: Erlotinib & a) NSCLC $[79,85]$ \\
\hline & & b) III & b) C: mFOLFOX6 & $\begin{array}{l}\text { b) HER2 negative(-) and c-Met+ } \\
\text { adenocarcinoma of the stomach or GEJ } \\
\text { (NCT01662869) }\end{array}$ \\
\hline Rilotumumab & HGF (c-Met ligand) MAb & III & Monotherapy & $\begin{array}{l}\text { c-Met+ gastric or GEJ adenocarcinoma } \\
\text { (NCT01697072) }\end{array}$ \\
\hline
\end{tabular}

Table 1: Inhibitors of EGFR/c-Met and their clinical status. Abbreviations: NSCLC: Non-small Cell Lung Cancer; HNSCC: Head and Neck Squamous Cell Carcinoma; CRPC: Castration-Resistant Prostate Cancer; CRC: Colorectal Cancer; HCC: Hepatocellular Carcinoma; GEJ: Gastroesophageal Junction; TKI: Tyrosine Kinase Inhibitor; MAb: Monoclonal antibody. ${ }^{\star}$ FDA approval was withdrawn in the USA.

\section{c-Met inhibitor clinical trials}

There are several ongoing clinical trials using c-Met inhibitors, which include both combinatorial and single drug therapies [78]. Many of these studies involve drugs that are in phase I clinical trials, in which the overall goal is to overcome acquired resistance. Phase II trials are also being conducted to improve PFS in various types of cancers [79-81]. Interesting results were obtained in a phase I clinical trial investigating the efficacy of a combinatorial therapy using tivantinib and sorafenib, a VEGF receptor inhibitor [82], in hepatocellular carcinoma (HCC) patients. In this trial, overall response rate (ORR) and disease control rate (DCR) were $10 \%$ and $70 \%$, respectively. In addition, median PFS were reported as 3.5 months, and successful responses included 1 complete response (CR), 1 partial response (PR), and 12 stable disease (SD) responses. Overall, this phase I trial found that the combination of tivantinib and sorafenib was well tolerated, and the observed antitumor activity of the combination therapy suggests that inhibition of c-Met along with other angiogenic modulators may have therapeutic potential, especially in patients already treated with VEGF receptor inhibitors [83].
In addition, a phase II trial with cabozantinib (XL-184) in castration-resistant prostate cancer (CRPC) patients showed promising results [80]. These results included regression of soft tissue tumors in $72 \%$ of patients and improved bone scans in $68 \%$ of evaluable patients, of which there was complete resolution of bone metastasis in $12 \%$ of patients after treatment with cabozantinib. After 12 weeks of the study, a 5\% ORR and SD in $75 \%$ of cabozantinib treated patients were also observed. In addition, median PFS was 23.9 weeks with cabozantinib treatment, compared to 5.9 weeks with only placebo treatment [80]. Other tumor types, such as ovarian cancer, are also currently being investigated in this clinical trial (NCT00940225) (Table 1).

\section{EGFR/c-MET Inhibitor Combination Clinical Trials}

As combinations of TKIs have proven to be more effective than single inhibitor treatment, EGFR and c-Met TKIs are currently being studied together in numerous clinical trials $[13,78]$. In a phase II clinical trial, NSCLC patients were given a combination of erlotinib and tivantinib. Tivantinib was shown to increase PFS from 9.7 to 16.1 weeks when given in combination with erlotinib. PFS improvement was particularly prominent among a subset of patients with nonsquamous histology, EGFR wild-type status, and k-RAS mutations 
[84]. A different phase II combinatorial study was conducted with 128 NSCLC patients, who had received at least one chemotherapy session, in which patients were given MetMAb (onartuzumab) intravenously along with erlotinib. Patients with high c-Met expression, $\geq 50 \%$ of tumor cells staining $2+$ or $3+$ intensity, were classified as "c-Met positive" by immunohistochemistry (IHC). These c-Met positive patients, who received both MetMAb and erlotinib had a reduced risk of disease progression, by $\sim 2$-fold, and a 3 -fold reduction in risk of death, as compared to erlotinib treatment alone [85]. More specifically, the combination treatment of MetMAb and erlotinib resulted in a $47 \%$ reduction in the risk of disease progression in c-Met positive patients, and the combination treatment significantly increased OS, compared to erlotinib treatment alone, 3.8 versus 12.6 months, respectively [79]. This benefit was not exclusive to EGFR mutation status or fluorescence in situ hybridization (FISH) analysis, but was predominately seen in patients with an IHC $2+$ or $3+$ status, suggesting that IHC is a more sensitive predictor of the benefit of MetMAb treatment $[79,85]$ (Table 1 ).

\section{Conclusion}

Receptor tyrosine kinases, EGFR and c-Met, and their corresponding ligands, EGF and HGF, play crucial roles for normal cell growth, development, differentiation, tissue migration, and wound healing. However, dysregulationof EGFR and c-Met in cancer cells, through gene amplification, overexpression, and mutational events, leads to increased malignancy of tumors and is associated with poor prognosis in a variety of cancers. Due to these malignant associations, EGFR and c-Met RTKs have been acknowledged as potential targets for cancer therapeutics, and have been extensively investigated. TKIs and MAbs targeting these receptors have proved to be effective in controlling the progression of tumors with high EGFR and c-Met expression. However, their use has been limited due to the development of resistance and subsequent recurrence of tumors. Therefore, the focus on EGFR and c-Met RTK inhibitors has shifted from studying their general mechanisms of action to investigating mechanisms of acquired resistance. Of importance, our laboratory has investigated different combinations of TKIs, targeting EGFR, c-Met, and proteins involved in downstream signaling, to explore possible EGFR/c-Met TKI mechanisms of resistance. Our results suggest that EGFR/c-Met TKI mechanisms of resistance may act through downstream signaling pathways, such as mTOR and Wnt, and that targeting of these pathways could be more effective in treatment of NSCLC patients [70]. In addition to pre-clinical studies, several clinical trials are ongoing in various tumor types, in which combinations of EGFR and c-Met inhibitors are being tested, in hope of improving anti-cancer effects and patient response. Further studies are required on this subject, as better understanding of the molecular mechanisms underlying drug resistance is necessary for the development of novel and effective cancer therapeutics.

\section{Acknowledgments}

Research reported in this publication was supported by National Cancer Institute of the National Institutes of Health under award number R21CA158965-01A1 http://www.nih.gov to Neelu Puri. The content is solely the responsibility of the authors and does not necessarily represent the official views of the National Institutes of Health. The funders had no role in study design, data collection and analysis, decision to publish, or preparation of the manuscript. The authors would like to acknowledge the Research Open Access
Publishing (ROAAP) Fund of the University of Illinois at Chicago for financial support towards the open access publishing fee for this article.

\section{References}

1. Siegel R, Ma J, Zou Z, Jemal A (2014) Cancer statistics, 2014. CA Cancer J Clin 64: 9-29.

2. Jemal A, Center MM, DeSantis C, Ward EM (2010) Global patterns of cancer incidence and mortality rates and trends. Cancer Epidemiol Biomarkers Prev 19: 1893-1907.

3. Ray M, Salgia R, Vokes EE (2009) The role of EGFR inhibition in the treatment of non-small cell lung cancer. Oncologist 14: 1116-1130.

4. Lawrence RE, Salgia R (2010) MET molecular mechanisms and therapies in lung cancer. Cell Adh Migr 4: 146-152.

5. Roskoski R Jr (2014) The ErbB/HER family of protein-tyrosine kinases and cancer. Pharmacol Res 79: 34-74.

6. Bazley LA, Gullick WJ (2005) The epidermal growth factor receptor family. Endocr Relat Cancer 12 Suppl 1: S17-27.

7. Bhardwaj V, Cascone T, Cortez MA, Amini A, Evans J, et al. (2013) Modulation of c-Met signaling and cellular sensitivity to radiation: potential implications for therapy. Cancer 119: 1768-1775.

8. Hemmings BA, Restuccia DF (2012) PI3K-PKB/Akt pathway. Cold Spring Harb Perspect Biol 4: a011189.

9. Leicht DT, Balan V, Kaplun A, Singh-Gupta V, Kaplun L, et al. (2007) Raf kinases: function, regulation and role in human cancer. Biochim Biophys Acta 1773: 1196-1212.

10. Roberts PJ, Der CJ (2007) Targeting the Raf-MEK-ERK mitogenactivated protein kinase cascade for the treatment of cancer. Oncogene 26: 3291-3310.

11. Pisick E, Jagadeesh S, Salgia R (2004) Receptor tyrosine kinases and inhibitors in lung cancer. ScientificWorldJournal 4: 589-604.

12. Cruz JJ, Ocaña A, Del Barco E, Pandiella A (2007) Targeting receptor tyrosine kinases and their signal transduction routes in head and neck cancer. Ann Oncol 18: 421-430.

13. Reungwetwattana T, Dy GK (2013) Targeted therapies in development for non-small cell lung cancer. J Carcinog 12: 22.

14. Sadiq AA, Salgia R (2013) MET as a possible target for non-small-cell lung cancer. J Clin Oncol 31: 1089-1096.

15. Lengyel E, Sawada K, Salgia R (2007) Tyrosine kinase mutations in human cancer. Curr Mol Med 7: 77-84.

16. Seshacharyulu P, Ponnusamy MP, Haridas D, Jain M, Ganti AK, et al. (2012) Targeting the EGFR signaling pathway in cancer therapy. Expert Opin Ther Targets 16: 15-31.

17. Ogiso H, Ishitani R, Nureki O, Fukai S, Yamanaka M, et al. (2002) Crystal structure of the complex of human epidermal growth factor and receptor extracellular domains. Cell 110: 775-787.

18. Yarden Y (2001) The EGFR family and its ligands in human cancer. signalling mechanisms and therapeutic opportunities. Eur J Cancer 37 Suppl 4: S3-8.

19. Harris RC, Chung E, Coffey RJ (2003) EGF receptor ligands. Exp Cell Res 284: 2-13.

20. Yarden Y, Sliwkowski MX (2001) Untangling the ErbB signalling network. Nat Rev Mol Cell Biol 2: 127-137.

21. Rocha-Lima CM, Soares HP, Raez LE, Singal R (2007) EGFR targeting of solid tumors. Cancer Control 14: 295-304.

22. Grandis JR, Sok JC (2004) Signaling through the epidermal growth factor receptor during the development of malignancy. Pharmacol Ther 102: $37-46$.

23. Li X, Shan BE, Wang J, Xing LP, Guo XJ, et al. (2013) Incidence and risk of treatment-related mortality with anti-epidermal growth factor receptor monoclonal antibody in cancer patients: a meta-analysis of 21 randomized controlled trials. PLoS One 8: e81897. 
24. Lurje G, Lenz HJ (2009) EGFR signaling and drug discovery. Oncology 77: 400-410.

25. Mendelsohn J, Baselga J (2006) Epidermal growth factor receptor targeting in cancer. Semin Oncol 33: 369-385.

26. Mendelsohn J, Baselga J (2003) Status of epidermal growth factor receptor antagonists in the biology and treatment of cancer. J Clin Oncol 21: $2787-2799$.

27. Graveel CR, Tolbert D, Vande Woude GF (2013) MET: a critical player in tumorigenesis and therapeutic target. Cold Spring Harb Perspect Biol 5 .

28. Koeppen H, Rost S, Yauch RL (2014) Developing biomarkers to predict benefit from HGF/MET pathway inhibitors. J Pathol 232: 210-218.

29. Kanteti R, Nallasura V, Loganathan S, Tretiakova M, Kroll T, et al. (2009) PAX5 is expressed in small-cell lung cancer and positively regulates cMet transcription. Lab Invest 89: 301-314.

30. Boon EM, van der Neut R, van de Wetering M, Clevers H, Pals ST (2002) Wnt signaling regulates expression of the receptor tyrosine kinase met in colorectal cancer. Cancer Res 62: 5126-5128.

31. Gazdar AF (2009) Activating and resistance mutations of EGFR in nonsmall-cell lung cancer: role in clinical response to EGFR tyrosine kinase inhibitors. Oncogene 28 Suppl 1: S24-31.

32. da Cunha Santos G, Shepherd FA, Tsao MS (2011) EGFR mutations and lung cancer. Annu Rev Pathol 6: 49-69.

33. Tang Z, Jiang S, Du R, Petri ET, El-Telbany A, et al. (2009) Disruption of the EGFR E884-R958 ion pair conserved in the human kinome differentially alters signaling and inhibitor sensitivity. Oncogene 28: 518-533.

34. Dienstmann R, De Dosso S, Felip E, Tabernero J (2012) Drug development to overcome resistance to EGFR inhibitors in lung and colorectal cancer. Mol Oncol 6: 15-26.

35. Kobayashi S, Boggon TJ, Dayaram T, Jänne PA, Kocher O, et al. (2005) EGFR mutation and resistance of non-small-cell lung cancer to gefitinib. N Engl J Med 352: 786-792.

36. Zhang Z, Lee JC, Lin L, Olivas V, Au V, et al. (2012) Activation of the AXL kinase causes resistance to EGFR-targeted therapy in lung cancer. Nat Genet 44: 852-860.

37. Sierra JR, Tsao MS (2011) c-MET as a potential therapeutic target and biomarker in cancer. Ther Adv Med Oncol 3: S21-35.

38. Sattler M, Reddy MM, Hasina R, Gangadhar T, Salgia R (2011) The role of the c-Met pathway in lung cancer and the potential for targeted therapy. Ther Adv Med Oncol 3: 171-184.

39. de Aguirre I, Salvatierra A, Font A, Mate JL, Perez M, et al. (2006) c-Met Mutational Analysis in the Sema and Juxtamembrane Domains in SmallCell-Lung-Cancer. Transl Oncogenomics 1: 11-18.

40. Neklason DW, Done MW, Sargent NR, Schwartz AG, Anton-Culver H, et al. (2011) Activating mutation in MET oncogene in familial colorectal cancer. BMC Cancer 11: 424.

41. Eder JP, Vande Woude GF, Boerner SA, LoRusso PM (2009) Novel therapeutic inhibitors of the c-Met signaling pathway in cancer. Clin Cancer Res 15: 2207-2214.

42. Hartmann JT, Haap M, Kopp HG, Lipp HP (2009) Tyrosine kinase inhibitors - a review on pharmacology, metabolism and side effects. Curr Drug Metab 10: 470-481.

43. Oldham RK, Dillman RO (2008) Monoclonal antibodies in cancer therapy: 25 years of progress. J Clin Oncol 26: 1774-1777.

44. Puri N, Salgia R (2008) Synergism of EGFR and c-Met pathways, crosstalk and inhibition, in non-small cell lung cancer. J Carcinog 7: 9 .

45. Sattler M, Abidoye O, Salgia R (2008) EGFR-targeted therapeutics: focus on SCCHN and NSCLC. ScientificWorldJournal 8: 909-919.

46. Keating GM (2014) Afatinib: a review of its use in the treatment of advanced non-small cell lung cancer. Drugs 74: 207-221.

47. Ou SH (2012) Second-generation irreversible epidermal growth factor receptor (EGFR) tyrosine kinase inhibitors (TKIs): a better mousetrap? A review of the clinical evidence. Crit Rev Oncol Hematol 83: 407-421.
48. Martinelli E, De Palma R, Orditura M, De Vita F, Ciardiello F (2009) Anti-epidermal growth factor receptor monoclonal antibodies in cancer therapy. Clin Exp Immunol 158: 1-9.

49. Cohen RB (2014) Current challenges and clinical investigations of epidermal growth factor receptor (EGFR)- and ErbB family-targeted agents in the treatment of head and neck squamous cell carcinoma (HNSCC). Cancer Treat Rev 40: 567-577.

50. Guan Z, Xu B, DeSilvio ML, Shen Z, Arpornwirat W, et al. (2013) Randomized trial of lapatinib versus placebo added to paclitaxel in the treatment of human epidermal growth factor receptor 2-overexpressing metastatic breast cancer. J Clin Oncol 31: 1947-1953.

51. Yamamoto N, Murakami H, Hayashi H, Fujisaka Y, Hirashima T, et al. (2013) CYP2C19 genotype-based phase I studies of a c-Met inhibitor tivantinib in combination with erlotinib, in advanced/metastatic nonsmall cell lung cancer. Br J Cancer 109: 2803-2809.

52. Elisei R, Schlumberger MJ, Müller SP, Schöffski P, Brose MS, et al. (2013) Cabozantinib in progressive medullary thyroid cancer. J Clin Oncol 31: 3639-3646.

53. Shah MA, Wainberg ZA, Catenacci DV, Hochster HS, Ford J, et al. (2013) Phase II study evaluating 2 dosing schedules of oral foretinib (GSK1363089), cMET/VEGFR2 inhibitor, in patients with metastatic gastric cancer. PLoS One 8: e54014.

54. Rodig SJ, Shapiro GI (2010) Crizotinib, a small-molecule dual inhibitor of the c-Met and ALK receptor tyrosine kinases. Curr Opin Investig Drugs 11: 1477-1490.

55. Munshi N, Jeay S, Li Y, Chen CR, France DS, et al. (2010) ARQ 197, a novel and selective inhibitor of the human c-Met receptor tyrosine kinase with antitumor activity. Mol Cancer Ther 9: 1544-1553.

56. Katayama R, Aoyama A, Yamori T, Qi J, Oh-hara T, et al. (2013) Cytotoxic activity of tivantinib (ARQ 197) is not due solely to c-MET inhibition. Cancer Res 73: 3087-3096.

57. Zhu K, Kong X, Zhao D, Liang Z, Luo C (2014) c-MET kinase inhibitors: a patent review (2011 - 2013). Expert Opin Ther Pat 24: 217-230.

58. Bendell JC, Ervin TJ, Gallinson D, Singh J, Wallace JA, et al. (2013) Treatment rationale and study design for a randomized, double-blind, placebo-controlled phase II study evaluating onartuzumab (MetMAb) in combination with bevacizumab plus mFOLFOX-6 in patients with previously untreated metastatic colorectal cancer. Clin Colorectal Cancer 12: 218-222.

59. Gordon MS, Sweeney CS, Mendelson DS, Eckhardt SG, Anderson A, et al. (2010) Safety, pharmacokinetics, and pharmacodynamics of AMG 102 , a fully human hepatocyte growth factor-neutralizing monoclonal antibody, in a first-in-human study of patients with advanced solid tumors. Clin Cancer Res 16: 699-710.

60. Wen PY, Schiff D, Cloughesy TF, Raizer JJ, Laterra J, et al. (2011) A phase II study evaluating the efficacy and safety of AMG 102 (rilotumumab) in patients with recurrent glioblastoma. Neuro Oncol 13: $437-446$.

61. Ryan CJ, Rosenthal M, Ng S, Alumkal J, Picus J, et al. (2013) Targeted MET inhibition in castration-resistant prostate cancer: a randomized phase II study and biomarker analysis with rilotumumab plus mitoxantrone and prednisone. Clin Cancer Res 19: 215-224.

62. Xu H, Stabile LP, Gubish CT, Gooding WE, Grandis JR, et al. (2011) Dual blockade of EGFR and c-Met abrogates redundant signaling and proliferation in head and neck carcinoma cells. Clin Cancer Res 17: 4425-4438.

63. Castoldi R, Ecker V, Wiehle L, Majety M, Busl-Schuller R, et al. (2013) A novel bispecific EGFR/Met antibody blocks tumor-promoting phenotypic effects induced by resistance to EGFR inhibition and has potent antitumor activity. Oncogene 32: 5593-5601.

64. Xu KP, Yu FS (2007) Cross talk between c-Met and epidermal growth factor receptor during retinal pigment epithelial wound healing. Invest Ophthalmol Vis Sci 48: 2242-2248.

65. Reznik TE, Sang Y, Ma Y, Abounader R, Rosen EM, et al. (2008) Transcription-dependent epidermal growth factor receptor activation by hepatocyte growth factor. Mol Cancer Res 6: 139-150. 
Citation: Stone A, Harrington K, Frakes M, Blank K, Rajanna S (2014) EGFR and c-Met Inhibitors are Effective in Reducing Tumorigenicity in Cancer. J Carcinog Mutagen 5: 173. doi:10.4172/2157-2518.1000173

Page 9 of 9

66. Spix JK, Chay EY, Block ER, Klarlund JK (2007) Hepatocyte growth factor induces epithelial cell motility through transactivation of the epidermal growth factor receptor. Exp Cell Res 313: 3319-3325.

67. Bean J, Brennan C, Shih JY, Riely G, Viale A, et al. (2007) MET amplification occurs with or without T790M mutations in EGFR mutant lung tumors with acquired resistance to gefitinib or erlotinib. Proc Natl Acad Sci U S A 104: 20932-20937.

68. Turke AB, Zejnullahu K, Wu YL, Song Y, Dias-Santagata D, et al. (2010) Preexistence and clonal selection of MET amplification in EGFR mutant NSCLC. Cancer Cell 17: 77-88.

69. Engelman JA, Zejnullahu K, Mitsudomi T, Song Y, Hyland C, et al. (2007) MET amplification leads to gefitinib resistance in lung cancer by activating ERBB3 signaling. Science 316: 1039-1043.

70. Fong JT, Jacobs RJ, Moravec DN, Uppada SB, Botting GM, et al. (2013) Alternative signaling pathways as potential therapeutic targets for overcoming EGFR and c-Met inhibitor resistance in non-small cell lung cancer. PLoS One 8: e78398.

71. Cruzalegui FH, Cano E, Treisman R (1999) ERK activation induces phosphorylation of Elk-1 at multiple S/T-P motifs to high stoichiometry. Oncogene 18: 7948-7957.

72. Acunzo M, Romano G, Palmieri D, Laganá A, Garofalo M, et al. (2013) Cross-talk between MET and EGFR in non-small cell lung cancer involves miR-27a and Sprouty2. Proc Natl Acad Sci U S A 110: 8573-8578.

73. Sutterlüty H, Mayer CE, Setinek U, Attems J, Ovtcharov S, et al. (2007) Down-regulation of Sprouty2 in non-small cell lung cancer contributes to tumor malignancy via extracellular signal-regulated kinase pathwaydependent and -independent mechanisms. Mol Cancer Res 5: 509-520.

74. Chong CR, Jänne PA (2013) The quest to overcome resistance to EGFRtargeted therapies in cancer. Nat Med 19: 1389-1400.

75. Moore MJ, Goldstein D, Hamm J, Figer A, Hecht JR, et al. (2007) Erlotinib plus gemcitabine compared with gemcitabine alone in patients with advanced pancreatic cancer: a phase III trial of the National Cancer Institute of Canada Clinical Trials Group. J Clin Oncol 25: 1960-1966.

76. Mitsudomi T, Morita S, Yatabe Y, Negoro S, Okamoto I, et al. (2010) Gefitinib versus cisplatin plus docetaxel in patients with non-small-cell lung cancer harbouring mutations of the epidermal growth factor receptor (WJTOG3405): an open label, randomised phase 3 trial. Lancet Oncol 11: 121-128.

77. Mitsudomi T, Morita S, Yatabe Y, Negoro S, Okamoto I, et al. (2012) Updated overall survival results of WJTOG 3405, a randomized phase III trial comparing gefitinib $(G)$ with cisplatin plus docetaxel $(C D)$ as the first-line treatment for patients with non-small cell lung cancer harboring mutations of the epidermal growth factor receptor (EGFR). J Clin Oncol 30 .

78. Yano S, Nakagawa T (2014) The current state of molecularly targeted drugs targeting HGF/Met. Jpn J Clin Oncol 44: 9-12.

79. Spigel DR, Ervin TJ, Ramlau RA, Daniel DB, Goldschmidt JH Jr, et al. (2013) Randomized phase II trial of Onartuzumab in combination with erlotinib in patients with advanced non-small-cell lung cancer. J Clin Oncol 31: 4105-4114.

80. Smith DC, Smith MR, Sweeney C, Elfiky AA, Logothetis C, et al. (2013) Cabozantinib in patients with advanced prostate cancer: results of a phase II randomized discontinuation trial. J Clin Oncol 31: 412-419.

81. Adjei AA, Schwartz B, Garmey E (2011) Early clinical development of ARQ 197, a selective, non-ATP-competitive inhibitor targeting MET tyrosine kinase for the treatment of advanced cancers. Oncologist 16: 788-799.

82. Wilhelm SM, Adnane L, Newell P, Villanueva A, Llovet JM, et al. (2008) Preclinical overview of sorafenib, a multikinase inhibitor that targets both Raf and VEGF and PDGF receptor tyrosine kinase signaling. Mol Cancer Ther 7: 3129-3140.

83. Martell RE, Puzanov I, Ma WW, Santoro A, Dy GK, et al. (2012) Safety and efficacy of MET inhibitor tivantinib (ARQ 197) combined with sorafenib in patients (pts) with hepatocellular carcinoma (HCC) from a phase I study. J Clin Oncol 30

84. Schiller JH, Akerley WL, Brugger W, Ferrari D, Garmey EG, et al. (2010) Results from ARQ 197-209: A global randomized placebo-controlled phase II clinical trial of erlotinib plus ARQ 197 versus erlotinib plus placebo in previously treated EGFR inhibitor-naive patients with locally advanced or metastatic non-small cell lung cancer (NSCLC). J Clin Oncol.

85. Spigel DR, Ervin TJ, Ramlau R, Daniel DB, Goldschmidt JH, et al. (2011) Final efficacy results from OAM4558g, a randomized phase II study evaluating MetMAb or placebo in combination with erlotinib in advanced NSCLC. J Clin Oncol 29

86. Pujol JL, Pirker R, Lynch TJ, Butts CA, Rosell R, et al. (2014) Metaanalysis of individual patient data from randomized trials of chemotherapy plus cetuximab as first-line treatment for advanced nonsmall cell lung cancer. Lung Cancer 83: 211-218.

87. Choueiri TK, Vaishampayan U, Rosenberg JE, Logan TF, Harzstark AL, et al. (2013) Phase II and biomarker study of the dual MET/VEGFR2 inhibitor foretinib in patients with papillary renal cell carcinoma. J Clin Oncol 31: 181-186. 